
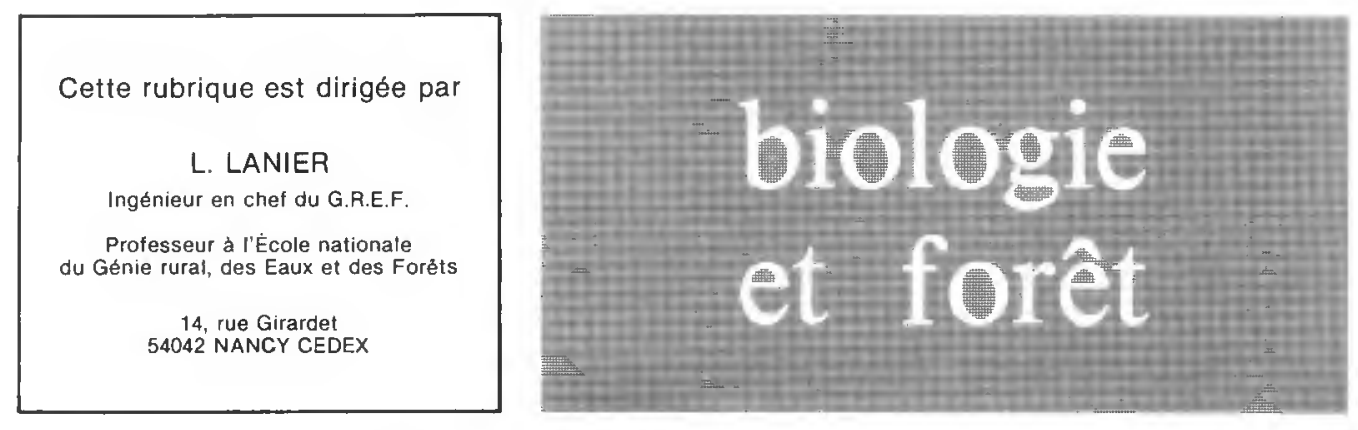

\title{
CLIMATOLOGIE DE L'ARBORETUM D'AMANCE (Meurthe-et-Moselle)
}

\author{
G. AUSSENAC - J.M. DESJEUNES - F. WILLM
}

En 1901, était créé un arboretum en bordure de la forêt d'Amance, près du village de Champenoux, à $13 \mathrm{~km}$ à l'est de Nancy. En installant cet arboretum, l'École nationale des Eaux et Forêts se pro. posait d'atteindre un double but : installer des collections d'arbres pour l'enseignement et étudier les espèces susceptibles d'être avantageusement utilisées pour l'enrichissement des forêts de l'Est de la France (Jolyet, 1908). Une étude sur l'arboretum d'Amance a été rédigée en 1961 par E.F. Debazac, qui décrit les conditions écologiques générales et dresse un catalogue des espèces. Des études particulières ont ensuite été réaliséès, citons les travaux de Debazac $(1965,1967)$ et d'Aussenac (1967).

Sans faire une description détaillée de l'arboretum, il est utile d'indiquer qu'il est situé à $250 \mathrm{~m}$ d'altitude et que les essences introduites relèvent de quatre grandes régions florales : l'Eurasie occidentale, l'Eurasie orientale, l'Amérique du Nord occidentale (versant de l'Océan Pacifique) et l'Amérique du Nord orientale (versant de l'Océan Atlantique) De 1936 à 1967, l'arboretum a été complété par la création de nouvelles plantations (Guinier, 1936). II convient d'ajouter que de nombreuses autres plantations expérimentales ont été faites en forêt d'Amance : comparaisons d'espẻces et de provenances, essais de densité de plantation, etc. En forêt domaniale d'Amance, l'essence dominante est le Chêne, rouvre ou pédonculé. Mais, le Hêtre y joue en mélange un rôle important, voire localement exclusif.

Les renseignements fournis par un arboretum ne peuvent être réellement valorisés que si l'on dispose diune description précise des conditions climatiques. Aussi, en 1951, un poste climatologique a été installé dans la pépinière voisine de cet arboretum. Par la suite, en 1966 et en 1975, les installations ont subi des moditications destinées à en améliorer le fonctionnement.

Comme l'écrit Godard (1951), la Lorraine, par sa situation de carrefour, voit se produire une "lutte " d'influences climatiques, d'autant plus difficile à saisir que le relief multiplie les nuances, 
crẻe des variètés en faisant apparaitre des contacts francs, et même brutaux, en favorisant certajnes pénétrations. Bien qu'appartenant aux climats continentaux, le climat lorrain est largement conditionné par les influences océaniques.

II nous a donc semblé intèressant d'analyser le climat local de l'arboretum d'Amance, de le situer dans le contexte régional et d'envisager les relations climat - production forestière en Lorraine.

\section{CARACTÉRISATION CLIMATIQUE DE L'ARBORETUM D'AMANCE}

Le poste climatologique a èté installè en 1951. II comportait un pluviomètre association et un abri météorologique type "Montsouris". En 1966, le poste a été rééquipé d'un abri type "Anglais", grand modèle, conforme au standard dèfini par la Météorologie nationale.

\section{Températures}

Afin de pouvoir effectuer des comparaisons valables avec les mesures faites par la Météorologie nationale, nous n'avons retenu que les mesures effectuées depuis 1967. Le tableau 1 donne les moyennes des températures maximum, minimum et moyenne pour la période 1967-1979. Les moyennes annuelles sont les suivantes:

- moyenne annuelle des maxima journaliers $: 13,4{ }^{\circ} \mathrm{C}$

- moyenne annuelle des minima journaliers : $4,8^{\circ} \mathrm{C}$

- temperature moyenne annuelle $\quad: 9,1^{\circ} \mathrm{C}$

Tableau 1 Données climatologiques mensuelles moyennes à l'arboretum d'Amance

\begin{tabular}{|c|c|c|c|c|c|c|}
\hline \multirow{2}{*}{ Mois } & \multicolumn{3}{|c|}{ Température $\left(\right.$ en $\left.^{\circ} \mathrm{C}\right)\left({ }^{1}\right)$} & \multirow{2}{*}{$\begin{array}{c}\text { Nombre } \\
\text { moyen } \\
\text { de jours } \\
\text { de gelée }\left({ }^{1}\right)\end{array}$} & \multirow{2}{*}{$\begin{array}{c}\text { Pluie } \\
\text { (mm) } \\
(2)\end{array}$} & \multirow{2}{*}{$\begin{array}{c}\text { Nombre } \\
\text { de jours } \\
\text { de pluie (2) }\end{array}$} \\
\hline & Max. & Min. & Moyenne & & & \\
\hline Janvier........ & 3,9 & $-\quad 1,2$ & 1,3 & 18,1 & 48,9 & 17 \\
\hline Février $\ldots \ldots \ldots$. & 5,8 & $-0,6$ & 2,6 & 16,0 & 50,9 & 15 \\
\hline Mars ......... & 9,3 & 1,0 & 5,2 & 12,6 & 47,2 & 14 \\
\hline Avril $\ldots \ldots \ldots$ & 13,2 & 3,2 & 8,2 & 7,9 & 44,9 & 13 \\
\hline Mai .......... & 17,7 & 7,2 & 12,4 & 0,7 & 64,1 & 15 \\
\hline Juin ...... & 21,0 & 10,2 & 15,6 & 0 & 71,8 & 12 \\
\hline Juillet . . . . . & 23,3 & 11,9 & 17,6 & 0 & 64,2 & 12 \\
\hline Août ......... & 22,6 & 11,8 & 17,2 & 0 & 73,2 & 13 \\
\hline Septembre..... & 19,4 & 8,8 & 14,1 & 0,1 & 58,9 & 15 \\
\hline Octobre ....... & 14,2 & 5,8 & 10,0 & 2,1 & 49,7 & 17 \\
\hline Novembre...... & 7,5 & 1,6 & 4,6 & 10,2 & 64,9 & 18 \\
\hline Décembre...... & 3,8 & $-1,0$ & 1,4 & 18,4 & 58,7 & 16 \\
\hline ANNÉE. & 13,4 & 4,8 & 9,1 & 86,1 & 697,4 & 177 \\
\hline
\end{tabular}

(1) Période 1967-1979 - (2) Période 1951-1979. 
Le mois le plus chaud est juillet, avec une température moyenne de $17,6^{\circ} \mathrm{C}$ et une moyenne des maxima de $23,3^{\circ} \mathrm{C}$.

Le mois le plus froid est janvier, avec une température moyenne de $1,3^{\circ} \mathrm{C}$ et une moyenne des minima de $-1,2^{\circ} \mathrm{C}$. Pendant la période considérée, les records absolus ont été : $-19,9^{\circ} \mathrm{C}$ le 13 janvier 1968 et $+35,2^{\circ} \mathrm{C}$ le 16 juillet 1976 .

D'une façon générale, on observe que, pendant la même période, les températures maximum et minimum sont légèrement plus faibles qu'au poste de la Météorologie nationale à NancyTomblaine. En effet, dans celte dernière station pendant la même période, la température moyenne a été de $9,5^{\circ} \mathrm{C}$. Cette différence s'explique en partie par une différence d'altitude de $50 \mathrm{~m}$ (200 m Nancy-Tomblaine et $250 \mathrm{~m}$ à l'arboretum); on admet en effet un gradient de $0,5^{\circ} \mathrm{C}$ par $100 \mathrm{~m}$, et par la proximité immédiate de l'agglomération nancéienne pour le poste de la Météorolo. gie nationale.

Il y a annuellement en moyenne 86 jours de gelées à l'arboretum d'Amance (tableau 1).

Sur la période 1967-1979, il a été possible de calculer le nombre moyen de jours où le sol a été gelé (tableau 2).

Tableau 2 Nombre moyen de jours où le sol était gelé à l'arboretum d'Amance

\begin{tabular}{|c|c|c|c|}
\hline \multirow{2}{*}{ Mois } & \multicolumn{3}{|c|}{ Profondeur dans le sol } \\
\hline & $-5 \mathrm{~cm}$ & $-10 \mathrm{~cm}$ & $-20 \mathrm{~cm}$ \\
\hline$\ldots \ldots \ldots \ldots \ldots \ldots \ldots$ & 18,0 & 7,3 & 1,7 \\
\hline Février.................... & 15,0 & 4,6 & 1,3 \\
\hline Mars $\ldots \ldots \ldots \ldots \ldots \ldots \ldots \ldots \ldots$ & 7,3 & 0,3 & 0,0 \\
\hline 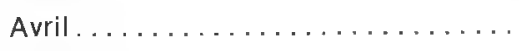 & 0,3 & 0,0 & 0,0 \\
\hline Septembre ................. & 0,0 & 0,0 & 0,0 \\
\hline Octobre....... & 0,1 & 0,0 & 0,0 \\
\hline Novembre..$\ldots \ldots \ldots \ldots \ldots \ldots$ & 1,7 & 0,0 & 0,0 \\
\hline Décembre ................... & 11,5 & 2,1 & 0,4 \\
\hline
\end{tabular}

La période 1967-1979 n'a pas été caractérisée par de grands hivers, aussi est-il intéressant de prendre en compte les données relevées par la Météorologie nationale pendant la période 1930-1960 à Nancy-Tomblaine (Garnier, 1967). Pendant cette période, les minima absolus pour les différents mois de l'année ont été les suivants :

$\begin{array}{llllll}\text { Janvier } & :-20,8{ }^{\circ} \mathrm{C} & \text { Mai } & :-4,2{ }^{\circ} \mathrm{C} & \text { Septembre : }-1,3{ }^{\circ} \mathrm{C} \\ \text { Février } & :-24,8{ }^{\circ} \mathrm{C} & \text { Juin } & : & 1,6{ }^{\circ} \mathrm{C} & \text { Octobre : }-7,9^{\circ} \mathrm{C} \\ \text { Mars } & :-13,3^{\circ} \mathrm{C} & \text { Juillet } & : 3,7{ }^{\circ} \mathrm{C} & \text { Novembre : }-9,7{ }^{\circ} \mathrm{C} \\ \text { Avril } & :-6,8{ }^{\circ} \mathrm{C} & \text { Août } & : & 3,9{ }^{\circ} \mathrm{C} & \text { Décermbre : }-21,3{ }^{\circ} \mathrm{C}\end{array}$

L'hiver 1961-1962 a été rigoureux, mais les minima absolus n'ont pas été inférieurs aux valeurs indiquées ci-dessus. Depuis la création de l'arboretum en 1901, on a noté des hivers très froids (Aussenac, 1975) : en 1916-1917, 1928-1929, 1938-1939, 1939-1940, 1940-1941, 1941-1942, 1944-1945, 1946-1947, 1955-1956, 1961-1962. II est probable que depuis le début du siècle, le minimum absolu de température a été atteint en février 1956 ; il a été vraisemblablement voisin de $-25^{\circ} \mathrm{C}$.

II faut noter que, en 1956, l'abaissement de température a été extrêmement rapide, $20^{\circ}$ entre le 30

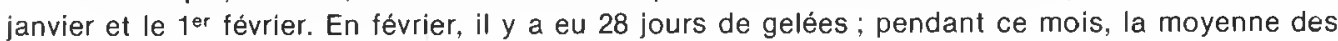


minima a été : $-15,2{ }^{\circ} \mathrm{C}$ et la moyenne des maxima : $-3,2^{\circ} \mathrm{C}$. Ainsi, 1956 s'est caractérisé par des températures basses, mais c'est surtout la durèe de cette période de froid qui est à prendre en considération, avec aussi la soudaineté du refroidissement. Il est certain que pour la végétation, un tel épisode climatique est beaucoup plus dommageable qu'un refroidissement progressif.

Les gelées occasionnent des dégâts importants lorsqu'elles affectent l'arbre durant sa période de croissance. A cet égard, deux types de gelées prèsentent des risques réels : les gelées tardives de printemps au moment du débourrement et les gelées précoces d'automne avant la fin de la crois. sance.

La fréquence des gelées tardives est une donnée essentielle pour caractériser une station. A Nancy (Aussenac, 1973), $5 \%$ des années prèsentent des risques de gelèe après le 21 mai. Mais cette frèquence des gelées doit être confrontée à l'état phénologique des arbres. En effet les dégâts de gelèe se produiront au moment du débourrement. Dans une étude effectuée en 1973, G. Aussenac a montrè que, à Nancy, des risques de dégâts de gelée tardive (1) existent 39 années sur 100 pour le Sapin pectiné, 22 années sur 100 pour le Douglas et 12 années sur 100 pour l'Epicéa commun.

Les gelées précoces peuvent aussi constituer un handicap sérieux surtout pour les essences exo. tiques à croissance longue. A Nancy, il géle $5 \%$ des annèes avant le 20 septembre et $26 \%$ des années avant le 10 octobre.

\section{Pluviomètrie}

La pluviométrie a été étudiée sur la période de 1951-1979. Le tableau 1 donne la pluviométrie mensuelle moyenne et le nombre moyen de jours de pluie. La pluviométrie annuelle moyenne à l'arboretum est de $697,4 \mathrm{~mm}$ répartis en 177 jours. Pendant la même période, les précipitations moyennes à Nancy-Tomblaine (Météorologie nationale) ont été de $728 \mathrm{~mm}$. Le tableau 3 permet de voir en 29 ans l'amplitude de variation de la pluviométrie : $467,6 \mathrm{~mm}$ en 1964 et $971,9 \mathrm{~mm}$ en 1979 . En ce qui concerne les valeurs mensuelles extrêmes, on notera en juin : $1,1 \mathrm{~mm}$ en 1962 et $189,4 \mathrm{~mm}$ en 1953 (tableau 4). Il est aussi intéressant de noter en 1976 une période de 32 jours sans précipitations (du 5 juin au 6 juillet).

Dans l'ensemble de l'année moyenne, les précipitations sont réparties de façon assez homogène. Le régime des pluies se caractérise par un léger maximum estival, ce qui indique un caractère continental peu marqué.

\section{Evapotranspiration potentielle et bilan hydrique}

La disponibilité en eau de la végétation forestière dépend de l'évapotranspiration potentielle qui définit le pouvoir évaporant de l'air et de l'offre hydrique au niveau du sol qui est fonction de la pluviométrie et de la réserve en eau du sol.

La réserve réellement utilisable par les arbres est difficile à évaluer. L'arboretum d'Amance est installé sur un sol à pseudogley se situant selon les cas à 50 ou $60 \mathrm{~cm}$ de profondeur. L'existence de cet horizon d'engorgement a pour conséquence l'existence d'une nappe perchée temporaire qui disparaît en été, mais qui constitue un supplément hydrique non négligeable que l'on peut estimer à $20 \mathrm{~mm}$. Les différentes mesures que nous avons effectuées à Amance nous conduisent alors à estimer la rèserve utilisable à $150 \mathrm{~mm}$.

(1) Le risque de dégàl est estimé par la controntation du risque de gelée (fréquence des annèes à gelée aprés une certaine date) et de la somme de température nécessaire pour atteindre le débourrement. La sommation des températures journaliéres moyennes est faite à partir du 1 er janvier, au-dessus du seuil de $2^{\circ} \mathrm{C}$. 
Biologie et forêt

Tableau 3 Precipitations annuelles et estivales de 1951 à 1979 à l'arboretum d'Amance

\begin{tabular}{|c|c|c|c|c|c|}
\hline \multirow{2}{*}{ Années } & \multicolumn{2}{|c|}{ Précipitations (mm) } & \multirow{2}{*}{ Années } & \multicolumn{2}{|c|}{ Prẻcipitations (mm) } \\
\hline & Annuelles & Estivales (1) & & Annuelles & Estivales (1) \\
\hline 1951 & 814 & 346 & 1966 & 824 & 242 \\
\hline 1952 & 911 & 242 & 1967 & 755 & 315 \\
\hline 1953 & 575 & 414 & 1968 & 778 & 371 \\
\hline 1954 & 590 & 264 & 1969 & 699 & 248 \\
\hline 1955 & 663 & 269 & 1970 & 935 & 306 \\
\hline 1956 & 580 & 284 & 1971 & 601 & 313 \\
\hline 1957 & 596 & 283 & 1972 & 672 & 289 \\
\hline 1958 & 837 & 289 & 1973 & 655 & 279 \\
\hline 1959 & 518 & 144 & 1974 & 793 & 283 \\
\hline 1960 & 798 & 364 & 1975 & 609 & 225 \\
\hline 1961 & 622 & 171 & 1976 & 480 & 178 \\
\hline 1962 & 518 & 180 & 1977 & 898 & 307 \\
\hline 1963 & 518 & 239 & 1978 & 763 & 256 \\
\hline 1964 & 467 & 181 & 1979 & 972 & 232 \\
\hline 1965 & 769 & 257 & & & \\
\hline
\end{tabular}

(1) Période : juin + juillet + août + septembre.

Tableau 4

Extrêmes mensuels et annuels de pluviométrie à l'arboretum d'Amance pendant la période 1951-1979

\begin{tabular}{|c|c|c|c|c|}
\hline \multirow{2}{*}{ Mois } & \multicolumn{2}{|c|}{ Minimum de pluviométrie } & \multicolumn{2}{|c|}{ Maximum de pluviométrie } \\
\hline & $\begin{array}{l}\text { Pluviométrie } \\
\text { (40 mm) }\end{array}$ & Annẻe & $\begin{array}{l}\text { Pluviométrie } \\
\text { (mm) }\end{array}$ & Année \\
\hline Janvier ......... & 14,0 & 1953 & 92,0 & 1955 \\
\hline Février ......... & 4,1 & 1959 & 142,4 & 1970 \\
\hline Mars.......... & 5,5 & 1961 & 115,0 & 1952 \\
\hline Avril .......... & 9,0 & 1955 & 87,0 & 1966 \\
\hline Mai ........... & 22,0 & 1953 & 145,9 & 1958 \\
\hline Juin . . . . . . . . & 1,1 & 1962 & 189,4 & 1953 \\
\hline Juillet ......... & 9,1 & 1979 & 158,3 & 1953 \\
\hline Août .......... & 12,9 & 1976 & 138,0 & 1972 \\
\hline Septembre...... & 11,3 & 1959 & 123,4 & 1967 \\
\hline Octobre...... & 3,4 & 1969 & 149,7 & 1974 \\
\hline Novembre....... & 13,8 & 1953 & 156,0 & 1952 \\
\hline Décembre... & 1,5 & 1963 & 156,3 & 1966 \\
\hline ANNÉE. & 467,6 & 1964 & 971,9 & 1979 \\
\hline
\end{tabular}




\section{G. AUSSENAC - J.M. DESJEUNES - F. WILLM}

Sur la base de cette réserve, et à partir des calculs d'évapotranspiration potentielle, le tableau 5 donne les déficits hydriques $\left({ }^{2}\right)$ pour les différentes années de 1967 à 1979.

On constate qu'il y a eu 6 années sur 13 qui ont présenté des déficits hycriques; la période 19741976 s'est caractérisée par des déficits importants. On voit ici que si 1976 a été une anriée de grande sécheresse, elle a été précédée de 2 années de sécheresse moindre, mais non négligeable.

\section{LE CLIMAT DE L'ARBORETUM D'AMANCE ET LES POTENTIALITÉS FORESTIĖRES}

Dans l'esprit des créateurs de l'arboretum, celui-ci était destiné à évaluer les possibilités de croissance d'un certain nombre d'espèces exotiques, susceptibles d'être utilisées en Lorraine.

Le tableau 6 rapporte les caractéristiques de quelques placettes de production de la station de Sylviculture et de Production situées en forêt domaniale d'Amance, à proximité de l'arboretum (Pardé, 1966, 1974 - Oswald et Pardé, 1976 - Oswald, 1980).

En forêt d'Amance, en 1976, un certain nombre d'espèces exotiques: Tsuga heterophy/la, Thuya plicata, Picea amorica ont été particulièrement sensibles à la sécheresse; par contre, Abies gran. dis et Pseudotsuga menziesii ont bien résisté, contirmant ainsi les résultats obtenus à partir d'étu. des écophysiologiques (Aussenac, 1978).

Des expériences mises en route au début du siècle à Amance, il faut retenir comme l'indique Pardé (1966) pour l'ensemble des essences introduites: pour les feuillus, le Chêne rouge d'Amérique (Quercus rubra) dont la production dépasse celle de notre Chêne local, et pour les résineux : le Douglas et le Sapin de Vancouver, dont les productions sont très élevées.

Dans quelle mesure les observations faites à Amance sur le comportement et la production des essences introduites sont-elles indicatrices pour la Lorraine? Les conditions écologiques (profondeur, richesse minimale, etc.) interviennent pour expliquer le comportement des arbres, mais l'analyse climatologique permet d'obtenir des indications générales.

L'arboretum d'Amance a une pluviométrie faible si on la compare à d'autres stations en Lorraine (figure 1). Cette pluviométrie limitée s'explique en grande partie par une altitude faible; en effet, ainsi qu'on peut le voir sur la figure 2, de faibles variations altitudinales s'accompagnent de variations notables de pluviométrie (figures 1 et 2 , page 36 ).

Les études effectuées (Aussenac et Ducrey, 1974 - Fontanel, 1979) au niveau du bilan hydrique en utilisant le rapport d'aridité de Thornthwaite (3) montrent que ce rapport est supérieur à $10 \%$ dans le nord-ouest de la Lorraine, alors qu'il est compris entre 0 et $10 \%$ dans l'ouest, le sud, l'est et le nord-est de la Lorraine, indiquant par là que les conditions d'alimentation hydrique sont excellentes dans la plus grande partie de cette région. On peut à cet égard conclure que, sur le plan hydrique, une grande partie de la Lorraine se trouve dans une situation plus favorable que l'arboretum d'Amance.

(2) Les déficits hydriques ont été calculés à partir d'un bilan hydrique établi à l'aide d'un modéle simple (Aussenac, Ducrey, 1974). L'évapotranspiration potentielle a été évaluée par la formule de Bouchet pour les mois de novembre à avril et la formule de Brochet. Gerbier pour les mois de mai à octobre.

(3) Rapport d'aridité de Thornthwaite : $\mathrm{R}_{\mathrm{a}}=\frac{\text { ETP }- \text { ETR }}{\text { ETP }} \cdot 100$, en prenant pour le calcul une réserve hydrique du sol $=100$ mm. 
Tableau 5

Evapotranspiration potentielle et déticits hydriques

pour des sols à réserve en eau de $150 \mathrm{~mm}$

\begin{tabular}{|c|r|r|r|}
\hline \multirow{2}{*}{ Année } & $\begin{array}{c}\text { Evapotranspiration } \\
\text { potentielle }(\mathrm{mm})\end{array}$ & $\mathrm{m}$ & \multicolumn{2}{|c|}{ Déficit hydrique } \\
\cline { 3 - 4 } & & période \\
\hline 1967 & 742,2 & 104,6 & juin à août \\
1968 & 628,7 & 0,0 & - \\
1969 & 608,0 & 20,5 & sept. - octobre \\
1970 & 620,2 & 0,0 & - \\
1971 & 619,5 & 0,0 & - \\
1972 & 610,5 & 0,0 & juillet et septembre \\
1973 & 638,0 & 55,6 & juin à août \\
1974 & 670,6 & 109,1 & juillet à septembre \\
1975 & 643,8 & 111,5 & mai à août \\
1976 & 751,3 & 317,1 & - \\
1977 & 620,8 & 0,0 & - \\
1978 & 599,3 & 0,0 & juillet \\
1979 & 609,1 & 6,4 &
\end{tabular}

Tableau 6

Caractéristiques dendrométriques de quelques placettes de production en forêt d'Amance

\begin{tabular}{|c|c|c|c|c|}
\hline Espèce & $\begin{array}{l}\text { Age } \\
\text { (ans) }\end{array}$ & $\begin{array}{l}\text { Hauteur } \\
\text { moyenne }(\mathrm{m})\end{array}$ & $\begin{array}{c}\text { Nombre } \\
\text { de tiges/ha }\end{array}$ & $\begin{array}{c}\text { Accroissement }\left({ }^{1}\right) \\
\text { moyen annuel } \\
(\mathrm{m} / \mathrm{ha} / \mathrm{an})\end{array}$ \\
\hline Abies nordmanniana...... & 59 & 22,3 & 397 & 6,0 \\
\hline Ables grandis........... & 47 & 27,3 & 807 & 17,6 \\
\hline Larix leptolepis....... & 59 & 23,0 & 380 & 8,0 \\
\hline Pinus strobus........... & 61 & 20,5 & 384 & 8,6 \\
\hline Pseudotsuga menziesii... & 27 & 18,9 & 1066 & 14,2 \\
\hline Quercus sessiliflora ( $\left.{ }^{2}\right)$. . & 100 & 22,3 & 400 & 4,4 \\
\hline Quercus rubra....... & 62 & 24,2 & 443 & 8,7 \\
\hline
\end{tabular}

(1) Bois fort tige.

(2) Espèce climacique focale de référence.

D'autres facteurs climatiques peuvent limiter la production de certaines espèces, c'est le cas des gelées tardives et des gelées prècoces.

Les gelées tardives résultent d'une configuration climatologique, en général homogène au niveau de la région, et très probablement les risques de dégâts sont du même ordre sur le Plateau Iorrain (4), sauf dans des situations microclimatiques particulières (fonds de vallon, plantations

(4) Par Plateau Iorrain, nous entendons la Lorraine à l'exception des côtes calcaires et des Vosges. 
enherbẻes, etc.). Une ètude faite dans le pays de Bitche (Guehl, 1978) montre que, dans cette région, par rapport à Nancy, il existe un retard du débourrement de près de deux semaines mais il n'y a pas une augmentation du risque de dégâts de gelée.

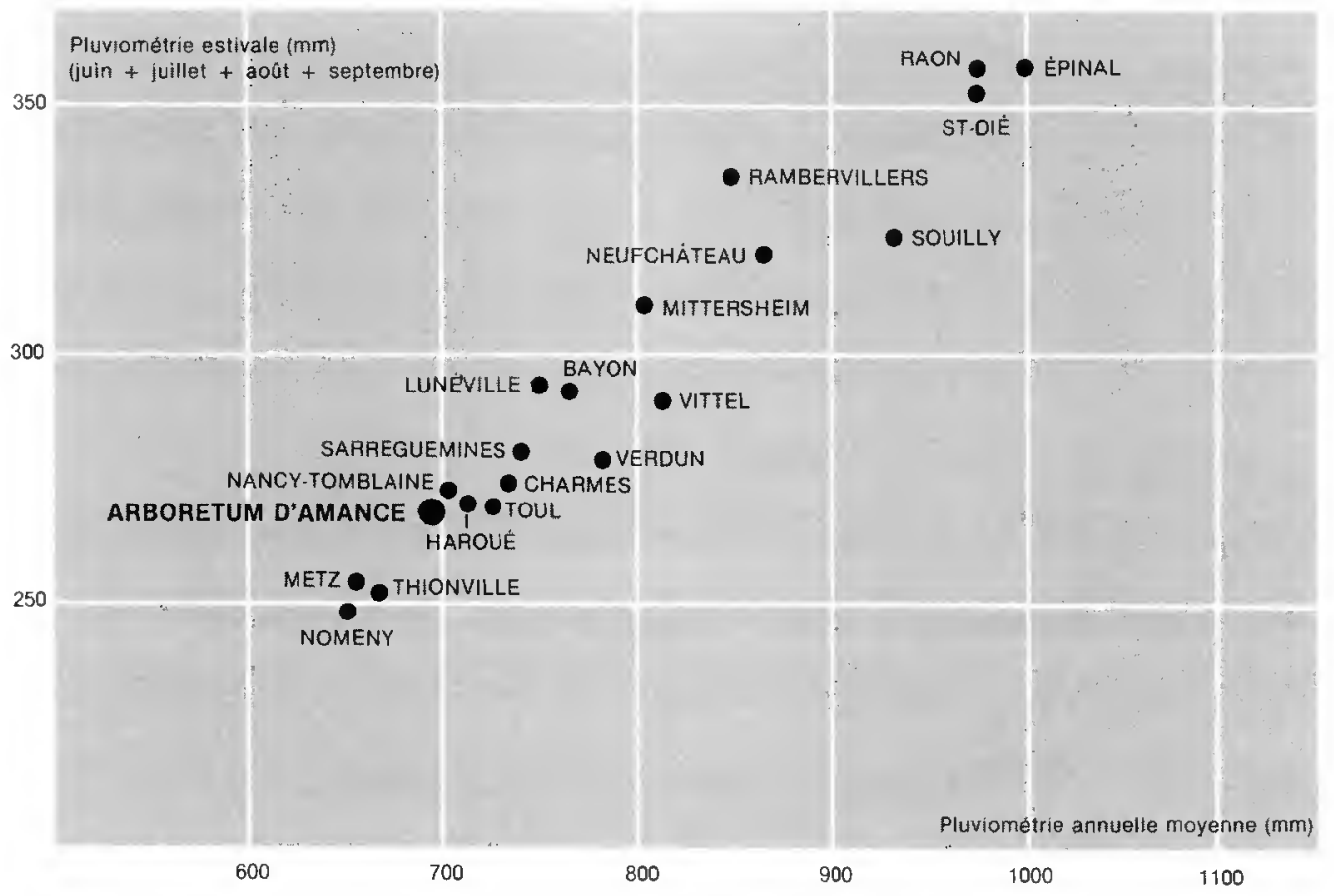

Figure 1. - DISTRIBUTION DE QUELQUES STATIONS PLUVIOMÉTRIQUES LORRAINES EN FONCTION DES PRÉCIPITATIONS ESTIVALES (juin + juillet + août + septembre) ET DE LA PLUVIOMÉTRIE ANNUELLE MOYENNE (période 1891-1930 pour l'ensemble des stations sauf pour l'arboretum d'Amance 1951-1979).

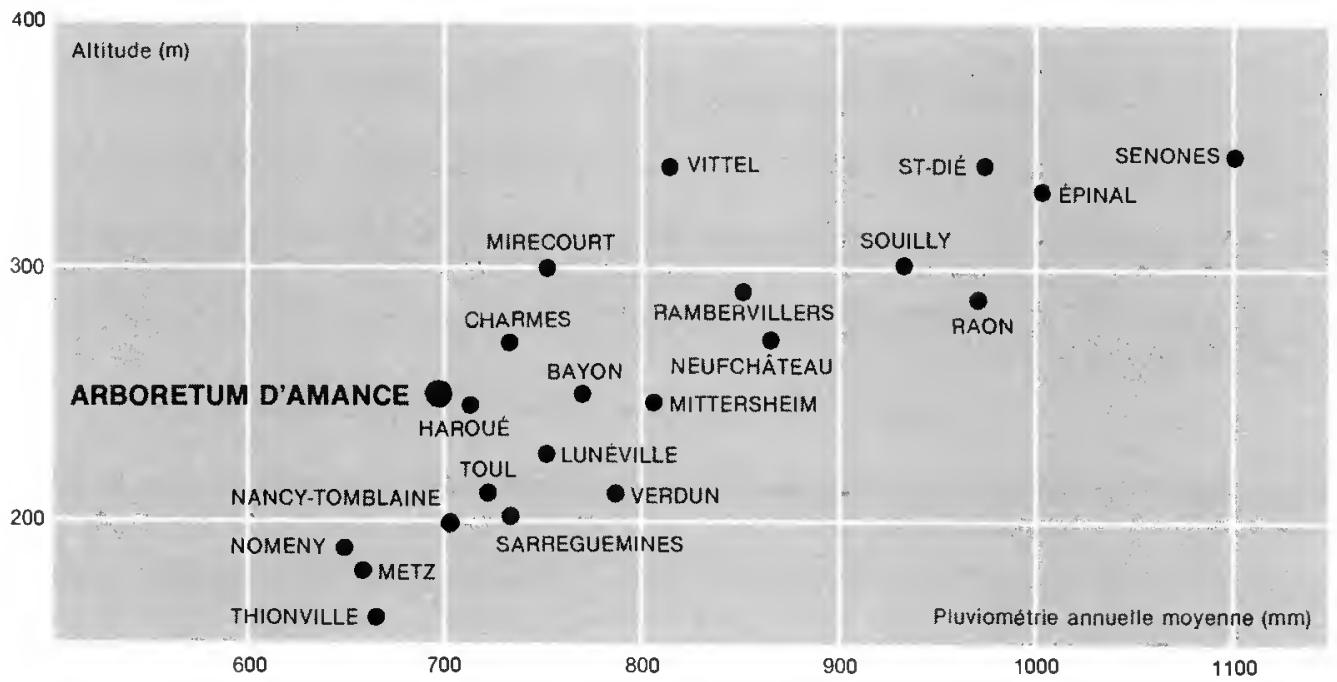

Figure 2. - OISTRIBUTION OE QUELDUES STATIONS PLUVIOMÉTHIQUES LORRAINES EN FONCTION DE L'ALTITUDE ET DE LA PLUVIOMÉTRIE ANNUELLE MOYENNE (période 1891-1930 pour toutes les stations saul pour l'arboretum d'Amance 1951-1979). 
On sait maintenant que la photosynthèse hivernale est possible chez les résineux, mais qu'elle est très fortement conditionnée par l'importance et le nombre de gelées. La précocité des gelées automnales a pour effet de réduire la période d'activité de l'arbre. II apparaît que, lorsqu'on s'éloigne vers l'est, le risque augmente; ainsi, à Bitche, il gèle dans $22 \%$ des cas avant le 20 septembre et dans $72 \%$ des cas avant le 10 octobre. II existe malheureusement très peu de données sur le plan thermométrique et il est difficile à cet égard d'avoir une description précise au niveau de la région.

En conclusion, l'étude climatique montre que les observations faites à partir de l'arboretum d'Amance et des plantations expérimentales environnantes sont représentatives des zones de basse altitude ( $200 \cdot 250 \mathrm{~m}$ ), mais sous-estiment probablement les possibilités forestières et bien d'autres situations du Plateau lorrain d'altitudes plus élevées.

Les arboretums ou les plantations comparatives d'espèces ou de provenance constituent un outil particulièrement efficace pour juger des possibilités d'utilisation d'espèces exotiques à utiliser dans une région considérée. Mais l'exploitation véritable des résultats obtenus ne peut être réalisée qu'en prenant en considération les conditions climatiques correspondant à la période d'étude. En effet tel ou tel résultat (dépérissement, mortalité, bonne croissance etc.) peut avoir pour explication des accidents ou des conditions climatiques normales dans la station considérée. Par ailleurs, la représentativité "climatique " du dispositif doit être précisée afin d'utiliser sans risques les résultats obtenus.

Les ètudes effectuées à partir des postes climatologiques de Detain Gergueil à propos de l'introduction du Sapin pectiné dans l'arrière côte bourguignonne (Ducrey, 1970), de l'arboretum de Royat pour l'étude des potentialités de production dans le Puy-de-Dôme (Aussenac-Ducrey, 1974), de la maison forestière du Hochkopf pour le Pays de Bitche (Guehl, 1978) montrent tout le parti que l'on peut tirer de telles études. II doit être possible d'aller plus loin dans l'utilisation de ce type d'étude en particulier dans les régions climatiquement mal connues.

En l'absence d'arboretum ou de plantations expérimentales locales, l'étude climatique seule peut permettre de préciser les possibilités d'utilisation de telle ou telle espèce et même d'en évaluer les potentialités de production.

C'est un fait bien connu de tous les forestiers que les postes climatologiques du réseau de la Météorologie nationale se situent tous dans des zones peu forestières. Or il existe, selon les régions, des gradients climatiques considérables et rapides dont on méconnaît l'importance et qui faussent l'interprétation des pertormances de'la forêt.

Grâce à d'importants progrès techniques récents, il est devenu possible de mettre en œuvre des postes climatologiques automatiques et autonomes. Pourquoi ne pas envisager maintenant l'installation de tels postes dans des zones à vocation torestière où existent de véritables "déserts " climatologiques?

La détermination des zones à équiper devrait répondre aux objectifs suivants :

- détermination des essences forestières susceptibles d'être utilisées,

- évaluation des capacités de production de ces mêmes essences (détermination des facteurs limitants).

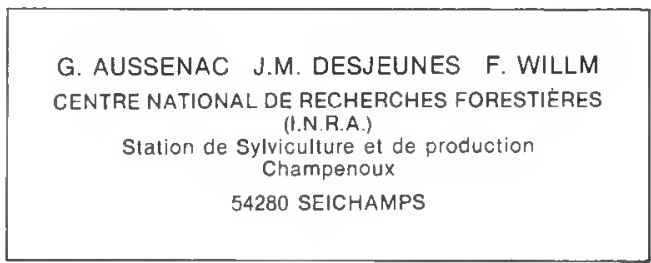




\section{BIBLIOGRAPHIE}

AUSSENAC (G.). - Observations sur la dissémination des graines chez Abies grandis. Revue forestière française $n^{\circ} 6,1966$, pp. 431-434.

AUSSENAC (G.). - Étude des gelées tardives en relation avec les problèmes de reboisement. Annales des Sciences Forestieres vol. 30, $\mathrm{n}^{\circ} 2,1973$, pp. 141-155.

AUSSENAC (G.), DUCREY (M.). - A propos de l'arboretum de Royat : climatologie et potentialités de production forestière. Revue forestiere française, $n^{\circ} 6,1974$, pp. 447-458.

AUSSENAC (G.). - Etude des relations climat-régénération naturelle du chêne dans la région de Nancy, de 1865 à 1972. Revue forestiere française, $\mathrm{n}^{\circ} 1,1975$, pp. 37-39.

AUSSENAC (G.). - Quelques données statistiques sur les premières gelées d'automne en France. Revue forestière française, $\mathrm{n}^{\circ} 6,1977$, pp. 452-456.

AUSSENAC (G.). - La sécheresse de 1976 : influences des déficits hydriques sur la croissance des arbres forestiers. Revue forestiere trançaise, $\mathrm{n}^{\circ} 2,1978$, pp. 103-114.

DEBAZAC (E.F.). - L'arboretum d'Amance (Meurthe-et-Moselle). Annales de l'École Nationale des Eaux et Forêts XVII, $n^{\circ} 3,1961$, pp. 339-345.

DEBAZAC (E.F.). - Observations sur le débourrement et la croissance en longueur de quelques résineux. Revue forestière française, $\mathrm{n}^{\circ} 2,1965, \mathrm{pp} .120-130$.

DEBAZAC (E.F.), CLAUDE (R.). - Nouvelles observations sur le débourrement et la croissance en longueur de quelques espèces de sapin. Revue forestiere française, $\mathrm{n}^{\circ} 3,1967, \mathrm{pp} .183-190$.

DUCREY (M.). - Conditions climatiques et introduction du Sapin pectiné (Abies pectina D.C.) dans l'ancienne côte bourguignonne. Revue forestiere française, $\mathrm{n}^{\circ}$ 6, 1970, pp. 619-626.

FONTANEL (J.-L.). - Etude des influences climatiques sur les potentialités de la production forestière. Mémoire E.N.I.T.E.F. - 1979. Document Station de Sylviculture et de Production - 74 p.

GARNIER (M.). - Climatologie de la France. Mémorial de la Météorologie, 1967, 294 p.

GODARD (A.). - Contribution à l'étude du climat lorrain. Revue de la Geographie de Lyon, n 3, 1951, pp. $297-310$.

GUEHL (J.-M.). - Climatologie du pays de Bitche. Données climatiques relevées à la Maison Forestière du Hochkopf. Document Station de Sylviculture et de Production, 1978 - $10 \mathrm{p}$.

GUINIER (Ph.). - L'arboretum de l'École nationale des Eaux et Forêts à Amance. Bulletin de la Société des Sciences de Nancy $n^{\circ} 7,8,1936, p p .136-146$.

JOLYET (A.). - Arboretum de l'Ecole nationale des Eaux et Forêts. Revue des Eaux et Forêts, $n^{\circ}$ 47, 1908, pp. 7079.

OSWALD (H.). - Une expérience d'espacement de plantation de Sapin de Vancouver (Abies grandis). Revue forestiere française, $\mathrm{n}^{\circ} 1,1980, \mathrm{pp} .60-79$.

OSWALD (H.), PARDÉ (J.). - Une expérience d'espacement de plantation de Douglas en torêt domaniale d'Amance. Revue forestière française, $n^{\circ} 3,1976$, pp. 185-192.

PARDÉ (J.). - Les plantations expérimentales de l'Etang de Brin en forêt domaniale d'Amance (Meurthe-etMoselle) (1901-1964). Revue forestière trançaise $n^{\circ} 2,1966, \mathrm{pp} .99-121$.

PARDÉ (J.). - La forêt domaniale d'Amance. Document Station de Sylviculture et de Production - $1974,10 \mathrm{p}$. 